\title{
Reemergence of measles in Bulgaria: a large outbreak in Plovdiv, 2010
}

\section{Dear Editor,}

Measles is a highly infectious airborne acute viral disease. In some of the cases it is accompanied by severe complications, which may result in death, including pneumonia and encephalitis. Each year more than 20 million people suffer from measles worldwide, predominantly in several parts of Africa and Asia, including the Eastern Mediterranean Region (EMR). ${ }^{1}$ Before the vaccination era, measles was widespread in the world. In the decade before the national measles vaccine program was implemented in the United States, it was estimated that about 4 million people had contracted measles. ${ }^{2}$ Out of approximately 500,000 measles cases reported annually, 500 resulted in death and 1,000 in permanent brain damage due to measles encephalitis. ${ }^{2}$

With the implementation of the routine measles immunization the incidence rate began to decrease progressively. This gave grounds to the World Health Organization (WHO) Regional Office for Europe to develop a program for the elimination of the disease. ${ }^{3}$ In Bulgaria, WHO case definitions and case classification of the European Union have been adopted since 2005..$^{-6}$

Despite the essential decrease of the morbidity in Europe in the last 4 years epidemic outbreaks of measles have been registered in many of the European countries: in France during 2008 - 604 cases; $^{7}$ in Ireland since August 2009-2010 - 320 cases; ${ }^{8}$ in Germany from March 15 until May 19, 2010 - 71 cases; ${ }^{9}$ in Poland for $2009-40$ cases. ${ }^{10}$ In Slovenia 2 cases of measles as nosocomial infections were registered in March 2010. ${ }^{11}$ In Greece from January to July 2010, 126 cases were announced originally related to the outbreak in Bulgaria. ${ }^{12}$ In Italy during the first six months of 2010,19 cases were confirmed in a population with consistently high and improving immunization coverage. ${ }^{13}$ In Spain from October 13 to December 7, 2010, 59 patients with measles were registered. ${ }^{14}$ In northern Serbia 121 cases were diagnosed with measles, 78 of which were laboratory-confirmed during $2007 .{ }^{15}$
In Bulgaria, measles immunization was introduced in $1969^{16,17}$ and in 1972 it became universal. Until 1982 the routine vaccination included one dose of measles vaccine administered in people $\geq 10$ months of age. During the period of 19831992, a two-dose schedule using monovalent measles vaccine was applied, firstly at 12 months and 4 years of age, and later at 12 and 24 months of age. In 1993, the measles, mumps, rubella (MMR) vaccine was introduced into the national vaccination schedule. Until 2000, the routine measles immunization consisted of the first dose with MMR vaccine given at 13 months of age and the second dose with monovalent measles vaccine at 12 years of age. Since 2001, a routine two-dose immunization with MMR vaccine has been implemented, administered at 13 months and 12 years of age.

The last indigenous cases of measles in Bulgaria were reported in 2001. ${ }^{17,18}$ From 2002 to 2008, only six measles cases have been registered, all of them imported: three from China (2005); one from Ukraine (2006); one from Germany (2007) and one from the United Kingdom (2008). ${ }^{19,20}$ After seven years without indigenous transmission of measles in Bulgaria, an increasing number of cases have been reported since April 15 2009. The epidemiological investigation revealed that the index case was imported in March of the same year from Germany. The patient, a 24-year-old man, became ill on March 12, four days after arrival from Hamburg, where he used to work. ${ }^{17}$ The subsequent three measles cases occurred among his close contacts (family members). They were laboratory-confirmed by the National Reference Laboratory in Sofia. The samples were then sent to the WHO Regional Reference Laboratory (RRL) for Measles and Rubella in Berlin for reconfirmation and measles virus (MV) genotyping. The nucleotide sequences of the variable part of measles virus $\mathrm{N}$ gene (450 nt) derived from these three cases were identical and classified as genotype D4. Their sequence is represented by the official WHO
Authors

Nikolay Todorov Vatev ${ }^{1}$ Mariana Vartigova Stoycheva ${ }^{2}$ Andrey Ivanov Petrov ${ }^{1}$

${ }^{1} \mathrm{MD}, \mathrm{PhD}$, Main Assistant Professors, Medical University Plovdiv, Bulgaria

${ }^{2}$ Professor, MD, DSc, Head of Department of Infectious Diseases, Parasitology and Tropical Medicine, Medical University Plovdiv, Bulgaria

Submitted on: 05/05/2011 Approved on: 05/13/2011

Correspondence to: Mariana Vartigova Stoycheva Medical University Department of Infectious Diseases, Parasitology and Tropical Medicine Vassil Aprilov 15a, 4002 Plovdiv, Bulgaria mariana_stoycheva@yahoo.com

We declare no conflict of interest.

(C2011 Elsevier Editora Ltda. All rights reserved. 
name MVs/Shumen.BGR/15.09/1(D4). ${ }^{17}$ Over the next few months of 2009 many indigenous cases of measles occurred and the outbreak covered the whole country.

The aim of the study was to analyze the epidemiological characteristics of the patients with measles treated in the Clinics of Infectious Diseases - University Hospital, Plovdiv during the first six months of 2010 .

\section{METHODS}

Measles is included in the notification system of infectious diseases in Bulgaria and any suspected measles case requires obligatory immediate notification (within 24 hours) by fax or phone from health centers, clinical settings or hospitals to the Regional Inspection for Prevention and Control of Public Health (RIPCPH). ${ }^{6}$ Notifications of measles cases are collected and analyzed nationally at the National Center for Infectious and Parasitic Diseases (NCIPD) in the Ministry of Health of Bulgaria. Measles cases are identified and classified using standard case definitions and case classifications. ${ }^{4,5} \mathrm{~A}$ confirmed case of measles is either laboratory confirmed or epidemiologically linked to a patient with laboratory-confirmed measles infection..$^{5}$ In Bulgaria, an outbreak of measles is defined as a chain of transmission with 3 or more confirmed cases. During the outbreak in Plovdiv in 2010, 417 (15\%) of all the cases were laboratory-confirmed by the National Reference Laboratory in Sofia and 876 (32\%) were linked epidemiologically with them. The laboratory confirmation was done through positive serological test for measles-specific IgM. The other cases (1481 - 53\%) were notified as probable. There was no practical opportunity for laboratory confirmation of those cases, as the epidemic situation expanded considerably and more than 15 cases were registered daily (sometimes reaching up to 40 a day) only in Plovdiv. These patients were diagnosed based on clinically manifested symptoms: fever (raised temperature of $38.3^{\circ} \mathrm{C}$ or higher), illness with generalized maculo-papular rash of 3 days duration or more, and cough, coryza, or conjunctivitis. All cases with complications (pneumonia or neurological symptoms) and those with atypical manifestation of clinical symptoms were laboratory confirmed.

Surveillance data for the studied patients include patients' household location, age, sex, ethnicity, measles vaccination status, history of contact with another measles case, date of rash appearance, date of patient's visit at health setting, date of investigation at patient's home, date of notification to the RIPCPH. Vaccination status of the cases was determined by parental or patient report and/or by review of the immunization card. The cases aged 14 months to 12 years were considered unvaccinated if they had not received the first dose MMR at 13 months and the patients older than 12 years - in cases in which they had received neither the second dose at 12 years nor both of the doses (respectively 13 months and 12 years).

We analyzed all patients with measles, hospitalized in the Clinics of Infectious Diseases - University Hospital, Plovdiv in 2010 after having received their informed consent.

\section{RESULTS}

In Bulgaria, a total of 21,984 cases of measles have been reported in 2010, and the cases from Plovdiv region were 2,774 (Figure 1). The incidence rate for the country was $289 / 100,000$ and for Plovdiv - 392/100,000.

From the patients in Plovdiv, 1,428 (51.5\%) were male. The incidence rate for male was $421 / 100,000$ and for female - 368/100,000. A total of 1,901 (69\%) of the patients in Plovdiv were hospitalized in the Clinics for Infectious Diseases - Medical University, Plovdiv. The median age of the hospitalized patients was 8 years (range 4 months to 63 years of age).

The distribution of the patients by months from January to June 2010 shows the dynamics of the outbreak. It began relatively smoothly with few cases during January and February (respectively $1.4 \%$ and $3.6 \%$ of all cases). They increased essentially in March (12.7\%) and reached their maximum in April (34.3\%) and May (29.3\%). In June a great decrease of the measles-cases was notified (18.6\%) and subsidence of the outbreak. During the following days (up to the middle of August) a few cases were registered, a total of 41 .

Most of the patients were hospitalized on epidemiological evidence, since most of them were representatives of the Roma ethnic group, who live in overpopulated households in bad hygienic conditions, and have more than 3 children. One of the hospitalized patients died (aged 4 months, with pneumonia) and 27 have been treated in the intensive care sector.

The analysis of the age distribution disclosed that the number of patients is the highest among children aged 13 months to 12 years (51\%).

This is the age group which includes the period from the vaccination with the first dose MMR (at 13 months) to the administration of the second dose (at 12 years). It was followed by the cases of infants aged 4 weeks to 13 months (27\%). The diseased from the elder age groups were in smaller numbers, respectively: $13-18$ years $(11 \%), 19-35$ years $(9 \%),>35$ years $(3 \%)$.

The age distribution by months did not show deviation from the age distribution of the case-patients for the whole period of the outbreak. There was no shifting of incidence of cases from one group to another.

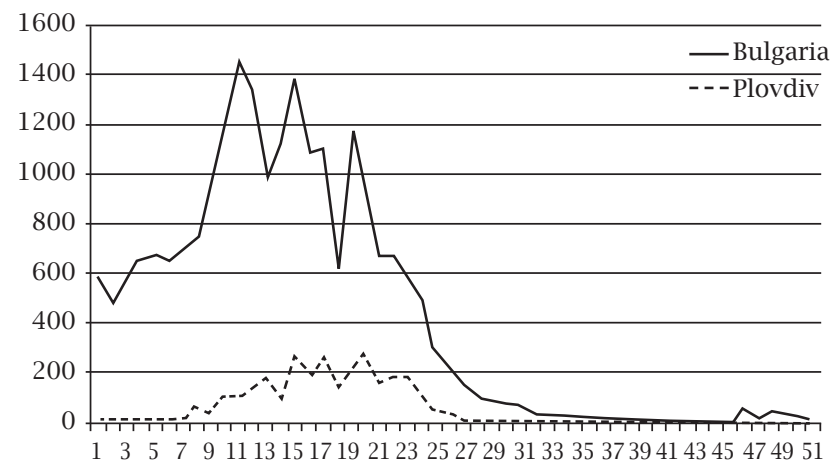

Figure 1: Registered measles cases by weeks in Bulgaria and Plovdiv - 2010. 
Most of the cases (59\%) occurred in individuals living in cities - probably due to the greater population density in cities which intensifies the epidemic process.

Significant difference was established between the occurrence of cases in the Roma population $(95.07 \%)$ and the Bulgarians (4.93\%). There were also significant differences in the age structure of both patient groups. Patients aged 13 months to 12 years (49\%) and infants (28\%) prevailed among Roma cases while among Bulgarians about $80 \%$ of the cases were above 18 years of age.

Most of the cases (65\%) were epidemiologically linked to another patient with measles and in $35 \%$ the initial case was not identified.

For $2 / 3$ of the cases $(64 \%)$ isolation was timely - on the first day after rash eruption or even during the last days of the catarrhal period. Cases of late isolation were not few in number $(36 \%)$ - they were isolated on the second or third day after rash eruption. The main reason for the delay was tardy seeking of medical attention and in some of the cases - delay in the transfer of patients from other clinics.

The study of the vaccination status (Table 1) showed that $33 \%$ of the patients had been vaccinated. Of the total of 892 patients aged 16 months to 12 years, $530(59 \%)$ were vaccinated. The percent of the vaccinated patients from the age group $13-18$ years was $32 \%$.

\section{DISCUSSION}

With the increasing vaccination coverage resulting from compulsory measles vaccination in 1972, the incidence of measles in Bulgaria was significantly reduced since 1977 . However, resurgences occurred in 1981-1982 and 1992. The resurgence in 1992 was attributed to lack of a second dose MMR at 12 years of age which was introduced at the end of $1992 .{ }^{16}$ The last resurgence began in April 2009. Until the end of 2010 a total of 24,233 cases had been notified with 24 deaths. The rapid resurgence could possibly be attributed to an accumulation of susceptible individuals (non-immune) to a critical threshold level. The outbreak arose in the Northeastern part of the country and gradually spread to all regions. From the notified cases for 2010 about 30,000 measles cases in Europe, ${ }^{21}$ the largest number was from Bulgaria - 21,766 (over 70\%). This large outbreak occurred while the data of NCIPD for the vaccination coverage against measles in Bulgaria was as follow: 2005: $1^{\text {st }}$ dose $-96 \%, 2^{\text {nd }}$ dose $-92 \% ; 2006: 1^{\text {st }}$ dose $-96 \%, 2^{\text {nd }}$ dose $-93 \% ; 2007: 1^{\text {st }}$ dose $-96 \%$, $2^{\text {nd }}$ dose $-94 \% ; 2008: 1^{\text {st }}$ dose $-96 \%$, 2nd dose $-94 \% .{ }^{17}$ The magnitude of the epidemic and the vaccination coverage showed discrepancy with the world practice for surveillance of the disease. According to practice with an immunization coverage of $>95 \%$, the outbreak of such a serious epidemic, that covers the whole country and the morbidity in 2010 reached 289/100,000 while in some regions it was above $600 / 100,000$, is impossible. This is probably due to improper recording and reporting of immunizations. This fact makes the surveillance of the disease difficult and can fully compromise the adopted National Program for the elimination of measles ${ }^{6}$ - what actually happened in practice. Measles is not only a preventable disease, it is also predictable. If the immunization coverage is recorded accurately, timely measures can be taken. When the immunization coverage is low, an additional vaccination campaign can be carried out before an epidemic outbreak.

The demographic characteristics of the cases show that measles is registered in over $95 \%$ among persons of Roma origin. While studies from other countries ${ }^{22}$ revealed that a considerable part (21\%) of the measles cases were among foreign visitors or imported from neighbor countries ${ }^{23}$ in Bulgaria the main risk group is the Roma population. The cause is the low vaccination coverage among them because of the intensive migration and the low health culture.

The analysis of the age distribution established high level (7\%) of the cases for patients aged $<6$ months. This is not in consonance with the data from other authors ${ }^{22,24} \mathrm{ac}$ cording to whom the relative part of the cases at this age is $1-4 \%$. Cases of measles among children $<6$ months are due to non-transmitted immunity from the mother to the child. Most of the mothers (> 90\%) from the Roma population are

Table 1. Vaccination coverage of the patients"

\begin{tabular}{|c|c|c|c|c|}
\hline Age group & Unvaccinated & Vaccinated & Unknown vaccination status & All \\
\hline$<6$ months & $139(100)$ & 0 & 0 & $139(7)$ \\
\hline $6-13$ months & $375(100)$ & 0 & 0 & $375(20)$ \\
\hline 14-16 months & $58(79)$ & $7(10)$ & $8(11)$ & $73(4)$ \\
\hline 17 months to 5 years & $91(29)$ & $190(61)$ & $32(10)$ & $313(16)$ \\
\hline 6 -12 years & $103(18)$ & $342(59)$ & $134(23)$ & $579(30)$ \\
\hline $13-18$ years & $78(39)$ & $65(32)$ & $59(29)$ & $202(11)$ \\
\hline 19-35 years & $41(25)$ & $28(17)$ & $93(58)$ & $162(9)$ \\
\hline$>35$ years & $43(74)$ & 0 & $15(26)$ & $58(3)$ \\
\hline Total & $928(49)$ & $632(33)$ & $341(18)$ & 1901 \\
\hline
\end{tabular}

*Data cover number of cases in age group (\% of age group with the vaccination status specified) or the total number of cases (\% of total for age group), unless otherwise indicated. 
aged 15-25 years. This implicitly means that in this age group there is also a pocket of non-vaccinated people. The part of the cases from 14 months to 12 years is too high - 51\%. The diseases at this age are due to omission of the 1st MMR dose at 13 months. Additionally, the cause for the high number of cases among people aged $13-18$ years (11\%) is omission of $2^{\text {nd }}$ MMR (or $1^{\text {st }}$ and $2^{\text {nd }}$ ) dose(s) at the age of 12 years.

Our observation on the proportion of measles cases in people over 19 years is not in agreement with previous reports that pointed out an age shift in measles incidence to older susceptible individuals. ${ }^{25,26}$ The percentage of cases over 19 years in our study is $12 \%$ but this shift of measles infection to adults is also disturbing as it has serious consequences for children. Infected adults could transmit measles virus to susceptible children. What is more, susceptible mothers cannot transmit protective anti-measles virus antibodies to newborn children, thus leaving them vulnerable to possible measles infection from their parents or other close contacts.

\section{CONCLUSION}

To prevent further outbreaks, surveillance should be enhanced to achieve complete coverage as well as consideration of older age group to avoid accumulation of susceptible persons. It is necessary to identify the areas with inadequate immunization performance, build up awareness among the hard-to-reach population to promote their vaccine-seeking behavior, repeat measles vaccination campaign to cover all children, conduct further community-based studies to identify the immunological status and reasons of vaccine failure. In addition, measles "catch-up" immunization campaigns should be conducted in Bulgaria to interrupt chains of transmission. Furthermore, the duration of impact of campaigns should not exceed $4-5$ years, even in best circumstances of high routine coverage. Implementing these measures among the Roma population in Bulgaria is of great importance because the routine immunization among these people is often compromised. Milestone for the elimination of measles is to achieve $>95 \%$ vaccination coverage with 2 doses MMR, but it has to be actual and not only existing on paper.

\section{REFERENCES}

1. World Health Organization, Geneva: World Health Organization, 2009. Measles. Available at: http://www.who.int/mediacentre/factsheets/fs286/en/index.html.

2. Strebel PM, Papania MJ, Dayan GH, et al. Measles vaccine. In: Plotkin SA, Orenstein WA, eds. Vaccines. Philadelphia: Elsevier, 2008:353-98.

3. World Health Organization, Regional Office for Europe. Strategic plan for measles and congenital rubella infection in the WHO European Region. Copenhagen, WHO Regional Office for Europe, 2003. Available from: http://www.euro.who.int/document/e81567.pdf

4. World Health Organization, Regional Office for Europe. Surveillance guidelines for measles and congenital rubella infection in the WHO European Region. Copenhagen, WHO Regional Office for Europe, 2003. Available from: http://www.euro.who.int/document/E82183.pdf

5. Commission Decision 2003/534/ EC amending Decision N2129/98/ EC of the European Parllament and of the Council and Decision 2000/96/ EC as regards communicable diseases listed in those Decisions and amending Decision 2002/253/ EC as regards the case defi- nitions for communicable diseases. Available from: http://eur-lex. europa.eu/LexUriServ/LexUriServ.do?uri=OJ:L2003:184:0035:00 39:EN:PDF

6. Ministry of Health of Bulgaria. Ordinance 21/18.07.2005 on the procedure for registration, notification and reporting of communicable diseases. State Gazette. 2005;62. Available from: http:// www.mh.government.bg/Articles.aspx?lang=bg-BG\&pageid=391 \&categoryid $=314 \&$ articleid $=552$

7. Waku-Kouomou D, Freymuth F, Parent du Chatelet I, et al. Cocirculation of measles virus genotypes during an epidemic in France in 2008. J Med Virol. 2010; 82:1033-43.

8. Gee S, Cotter S, O Flanagan D. Spotlight on measles 2010: measles outbreak in Ireland. Euro Surveill. 2010; 15(9):19500-03.

9. Roggendorf H, Mankertz A, Kundt R, et al. Spotlight on measles 2010: measles outbreak in a mainly unvaccinated community in Essen, Germany, March-June 2010. Euro Surveill. 2010; 15(26):19605-07.

10. Ortikova H, Rogalska J, Kazanovska-Zielinska E, et al. Spotlight on measles 2010: a measles outbreak in a Roma population in Pulawy, eastern Poland, June to August 2009. Euro Surveill. 2010; 15(17):19550-54.

11. Siegfried N, Wlisong C, Pienaar D. Too little, too late: measles epidemic in South Africa. Lancet. 2010; 376(9736):160.

12. Pervanidu D, Horefti E, Patrinos S, et al. Spotlight on measles 2010: ongoing measles outbreak in Greece, January - July 2010. Euro Surveill. 2010;15(30):19629.

13. Cova M, Cucchi A, Turla G, et al. Spotlight on measles 2010: in creased measles transmition in Ferrara, Italy, despite high vaccination coverage, March to May 2010. Euro Surveill. 2010; 15(50):19747.

14. López Hernández B, Laguna Sorinas J, Marín Rodríguez I, et al. Spotlight on measles 2010: an ongoing outbreak of measles in an unvaccinated population in Granada, Spain, October to November 2010. Euro Surveill. 2010; 15(50):19746.

15. Seguliev Z, Đurić P, Petrovic V, et al. Current measles outbreak in Serbia: a preliminary report. Euro Surveill. 2007; 12(11):3155.

16. Mihailov A, Mihneva Z. [Specific immunoprophylaxis for measles in Bulgaria]. Information Journal NCIPD. 2004; 6:36-40.

17. Marinova L, Kojouharova M, Mihneva Z. An ongoing measles outbreak in Bulgaria, 2009. Euro Surveill. 2009; 14(26):19259.

18. Gacheva N, Kojouharova M, Vladimirova N, et al. Acute infectious diseases in Bulgaria in 2001. Analysis of the main epidemiological indicators. Information Journal NCIPD. 2002; 40(5).

19. Kojouharova M, Vladimirova N, Kurchativa A, et al. Acute infectious diseases in Bulgaria in 2005-2006 (main epidemiological indicators). Information Journal NCIPD. 2008.51(4-5).

20. Kojouharova M, Kurchativa A, Vladimirova N, et al. Acute infectious diseases in Bulgaria in 2007 (main epidemiological indicators). Information Journal NCIPD. 2008;40(6).

21. European Centre for Disease Prevention and Control (ECDC). Epidemiological update on measles in EU/EEA. Stockholm: ECDC. 31 Mar 2011. Available from: http://www. ecdc.europa.eu/en/activities/sciadvice/Lists/ECDC Reviews/ ECDC_DispForm.aspx? List $=512 \mathrm{ff} 74 \mathrm{f}-77 \mathrm{~d} 4-4 \mathrm{ad} 8-\mathrm{b} 6 \mathrm{~d} 6$ bf0f23083f30\&ID=1046\&Source=http://www.ecdc.europa.eu/en/ activities/sciadvice/Lists/ECDC\%20Reviews/all\%20items.aspx

22. Fiebelkorn AP, Redd SB, Gallagher K, et al. Measles in the United States during the Postelimination Era. J I D. 2010; 202:1520-8.

23. Nmor JC, Thanh HT, Goto K. Recurring measles epidemic in Vietnam 2005-2009: implication for strengthened control strategies. Int J Biol Sci. 2011; 7:138-46

24. Al-Arabi Al-Ghamdi AM, Al-Farash MH, Ibrahim HM, et al. Epidemiological analysis of notified measles cases and review of surveillance system during an outbreak in Tabuk, Saudi Arabia. Ann Epidemiol. 2011; 21:262-71.

25. Nanyunja M, Lewis RF, Makumbi I, et al. Impact of mass measles campaign among children less than 5 years old in Uganda. J Infect Dis. 2003; 187(1):63-8.

26. Duclas P, Redd SC, Varuglese P, et al. Measles in adults in Canada and the United States: Implications for measles elimination and eradication. Int J Epidemiol.1999; 28(1):141-6 